\title{
Effectiveness and safety of a prehospital program of continuous positive airway pressure (CPAP) in an urban setting
}

\author{
Andrew Willmore, $\mathrm{MD}^{*{ }^{\dagger}}$; Richard Dionne, $\mathrm{MD}^{*{ }^{\dagger}}$; Justin Maloney, $\mathrm{MD}^{*{ }^{\dagger}}$; Ed Ouston, $\mathrm{ACP}^{\ddagger}$; Ian Stiell, \\ $\mathrm{MD}, \mathrm{MSc}^{* \S}$
}

\section{ABSTRACT}

Background: Continuous positive airway pressure (CPAP) is commonly used in the treatment of acute cardiogenic pulmonary edema (ACPE) and acute exacerbations of chronic obstructive pulmonary disease (AECOPD). In-hospital evidence is robust: CPAP has been shown to improve respiratory status and to reduce intubation rates. There is less evidence on prehospital CPAP, although the emergency medical services (EMS) adoption of this modality is increasing. The objectives of this study were to 1) measure the effectiveness of prehospital CPAP on morbidity, mortality, and transport times; and 2) audit the selection of patients by medics for appropriateness and safety.

Methods: We conducted a before-and-after study from August 1 to October 31 in 2010 and 2011, before and after the implementation of prehospital CPAP in a city of one million people with large rural areas. Medics were trained to apply CPAP to patients with respiratory distress and a presumed diagnosis of ACPE or AECOPD. Charts were selected using the search criteria of the chief complaint of shortness of breath, emergent transport to hospital, and any patients receiving CPAP in the field. Data extracted from ambulance call reports and hospital records were analysed with appropriate univariate statistics.

Results: A total of 373 patients enrolled (186 pre-non-invasive ventilation [NIV] and 187 post-NIV), mean age 71.5 years, female $51.4 \%$, and final diagnoses of ACPE $18.9 \%$, AECOPD $21.9 \%$. In the post group of 84 patients meeting NIV criteria, $41.6 \%$ received NIV; and of 102 patients not meeting the criteria, $5.2 \%$ received NIV. There were 12 minor adverse events in 36 applications (33.3\%) as per protocol. Comparing post versus pre, there were higher rates of emergency department (ED) NIV $(20.0 \%$ v. $13.4 \%, p<0.0001)$ and higher overall mortality $(18.8 \%$ v. $14.9 \%, p<0.0001)$. There were no differences in ED intubation $(2.1 \%$ v. $2.3 \%, p<0.001)$ and length of stay (6.8 v. 8.7 days, $p=0.24$ ).

Conclusion: Despite the robust in-hospital data supporting its use, we could not find benefit from CPAP in our prehospital setting with respect to morbidity, mortality, and length of stay. EMS must exercise caution in making the decision to invest in the equipment and training required to implement prehospital CPAP.

\section{RÉSUMÉ}

Contexte: La ventilation spontanée en pression positive continue (VSPPC) est souvent utilisée dans le traitement de I'œdème aigu du poumon cardiogénique (OAPC) et des exacerbations de bronchopneumopathie chronique obstructive (BPCO). En milieu hospitalier, les données sont claires: la VSPPC améliore l'état respiratoire des patients et abaisse les taux d'intubation; toutefois, il existe moins de données sur la VSPPC en milieu préhospitalier, bien que les services médicaux d'urgence y recourent de plus en plus. L'étude avait donc pour objectifs de: 1) mesurer l'efficacité de la VSPPC préhospitalière sur la morbidité, la mortalité et la durée de transport; 2) vérifier la pertinence du choix des patients par les ambulanciers paramédicaux et l'innocuité de l'intervention.

Méthode: Une étude de type prétest/post-test a été menée du $1^{\mathrm{er}}$ août au 31 octobre en 2010 et en 2011, soit avant et après la mise en œuvre de la VSPPC préhospitalière dans une ville de un million de personnes, entourée de grandes régions rurales. Les ambulanciers ont reçu une formation sur l'application de la VSPPC chez les patients en détresse respiratoire et chez ceux qui souffraient d'OAPC ou d'exacerbations de BPCO présumés. La sélection des dossiers s'est faite à l'aide des critères de recherche suivants, soit l'essoufflement comme principal motif d'appel, le transport d'urgence à l'hôpital et l'application de la VSPPC sur place, quel qu'en soit le motif. Les données extraites des rapports de sortie d'ambulance et les dossiers d'hôpitaux ont été analysés à l'aide de statistiques à une variable, appropriées. Les auteurs ont constitué un échantillon de commodité

Résultats: Ont été sélectionnés 373 patients (186 soumis à la ventilation non effractive [VNE] durant la phase "prétest " et 187 soumis à la VNE durant la phase " post-test ") ayant les caractéristiques suivantes: âge moyen: 71,5 ans; femmes: $51,4 \%$;

From the *Department of Emergency Medicine, University of Ottawa, Ottawa, ON.; †Regional Paramedic Program for Eastern Ontario, Ottawa Hospital, Ottawa, ON; ‡Ottawa Paramedic Service, Ottawa, ON; and §Ottawa Hospital Research Institute, Ottawa, ON.

Correspondence to: Andrew Willmore, The Ottawa Hospital General Campus, Department of Emergency Medicine, 501 Smyth Road, Ottawa, ON K1H 8L6; Email: awillmore@toh.on.ca 
diagnostic définitif: OAPC, 18,9\%; exacerbations de BPCO, $21,9 \%$. Dans le groupe "post-test " comptant 84 patients qui satisfaisaient aux critères de VNE, $41,6 \%$ y ont été soumis; et sur 102 patients qui ne répondaient pas aux critères, 5,2\% ont été soumis à la VNE. II s'est produit 12 événements défavorables mineurs dans 36 interventions (33,3\%) appliquées selon le protocole, ce qui laisse supposer l'absence de problèmes de sécurité. Après avoir comparé les résultats "prétest " avec les résultats " post-test ", les auteurs ont constaté qu'il y avait des taux plus élevés de VNE au service des urgences (SU) $(13,4 \%$ avant contre [c.] $20 \%$ après; $p<0,0001)$ et de mortalité générale $(18,8 \%$ c. $14,9 \%$; $p<0,0001)$; par contre, il n'y avait pas d'écart pour ce qui est de l'intubation au SU $(2,1$ c. $2,3 \% ; p<0,001)$ ou de la durée de séjour $(6,8$ c. 8,7 jours; $p=0,24)$. Après comparaison de tous les patients admissibles à la VSPPC dans les deux groupes, il ne s'est pas dégagé d'écart significatif en ce qui concerne la mortalité.

Conclusion: Malgré les données solides, recueillies dans les hôpitaux et les revues systématiques qui étayent l'application de la VSPPC en milieu intra- et extra-hospitalier, les auteurs de l'étude n'ont pas pu démontrer les avantages de la VSPPC en milieu préhospitalier au regard de la morbidité, de la mortalité et de la durée de séjour, pas plus qu'ils n'ont cerné de problème de sécurité lié à l'application de la VSPPC. Ce sont dans les comparaisons des seuls patients admissibles à la VSPPC, tous espaces de temps confondus, qu'il n'y avait pas d'écart de mortalité. Par ailleurs, $47 \%$ seulement des patients admissibles à la VSPPC ont été soumis à l'intervention appliquée selon le protocole, et $5,4 \%$ des patients inadmissibles $\mathrm{y}$ ont été soumis, ce qui donne à penser que la mise en œuvre du programme dans la cohorte " post-test " était incomplète. L'étude décrite ici vient grossir le nombre de petites études observationnelles de mise en œuvre de la VSPPC en milieu préhospitalier.

Keywords: chronic obstructive pulmonary disease (COPD), continuous positive airway pressure (CPAP), emergency medical services (EMS), non-invasive, non-invasive ventilation (NIV), prehospital, ventilation

\section{INTRODUCTION}

Respiratory distress secondary to acute cardiogenic pulmonary edema (ACPE) and acute exacerbations of chronic obstructive pulmonary disease (AECOPD) are common presenting complaints for emergency medical services (EMS). Until recently, the main prehospital interventions that have specifically targeted these disease processes are supplemental oxygen, bronchodilators, nitrates, and, in severe cases, assisted ventilation with bag valve mask (BVM) or endotracheal intubation. ${ }^{1,2}$ Some services use furosemide as a diuretic and morphine at low doses as an anxiolytic, but these are controversial and have been removed from many protocols. ${ }^{3}$

Continuous positive airway pressure (CPAP) is a form of non-invasive ventilation (NIV) that has been demonstrated to be a useful adjunct in the management of ACPE and various forms of respiratory failure in the hospital setting. ${ }^{4-6}$ As with other forms of NIV, CPAP results in faster clinical improvement and can reduce the requirement for invasive ventilation that increases the risk of complications, including nosocomial infections (pneumonia, sinusitis) and tracheal injury, which prolong intensive care unit (ICU) stay and hospital stay. $^{2,7,8}$ A growing body of research has recently explored the application of this intervention in the prehospital setting, and there is some evidence to support a benefit in regard to morbidity, mortality, and cost. $^{2,6,9}$ Barriers to the early adoption of prehospital CPAP were largely due to limitations in technology, money, and training. Although the adoption of this modality has increased to include most systems, the overwhelming management for these diseases has been the use of symptom relief drugs and traditional ventilatory support using high-flow demand oxygen devices (non-rebreather) and ventilatory assistance using BVM. There have been significant advances in producing cost-effective portable CPAP devices, but the implementation of this technology still requires a significant allocation of resources. As such, ongoing research is needed to determine the effectiveness and safety of this prehospital intervention on patient outcomes.

The objectives of this study were to 1 ) determine the effectiveness of appropriately administered prehospital CPAP on specific patient outcomes of morbidity and mortality, and 2) determine the ability of paramedics to adequately identify and safely treat patients with CPAP in a newly implemented prehospital protocol.

\section{METHODS}

\section{Design and setting}

A before-and-after study was conducted from August 1 to October 31 in 2010 and in 2011, before and after the implementation of prehospital CPAP in a city of one million people with large rural areas. The study size and period were chosen due to pragmatic constraints of budget and time. We compared the same 3-month period in 2 successive years to minimize bias from 
seasonal variations in disease prevalence. Almost all ambulances in this region are assigned one primary (i.e., basic life support) and one advanced care (i.e., advanced life support) paramedic. This study was approved by the Research Ethics Board of The Ottawa Hospital.

\section{Study population}

The study population was composed of persons ages 12 years and older and at least $40 \mathrm{~kg}$ of body weight with onset of respiratory distress assessed as ACPE or AECOPD by paramedics. All participants were transported by ambulance and treated in two emergency departments (EDs) in eastern Ontario.

\section{Study intervention}

Paramedics were trained to apply CPAP to patients with respiratory distress and a presumed diagnosis of ACPE or AECOPD prior to the commencement of the after period. Paramedics were trained with a 2-hour classroom module that included hands-on training in the ambulance. There was a follow-up video presentation (online) and continuing medical education review module. The device used was the Pulmodyne O2RESQ System, which delivers $30 \% \mathrm{FiO}_{2}$. CPAP could be applied at $5 \mathrm{~cm} \mathrm{H}_{2} \mathrm{O}$ to $15 \mathrm{~cm} \mathrm{H}_{2} \mathrm{O}$.

We allotted a 3-month run-in period to allow for initial adoption issues to be sorted out before collecting data. Criteria for CPAP application were 1) at least two of the following: respiratory rate $\geq 24$ breaths per minute, oxygen saturation $<90 \%$, and accessory muscle use; 2) paramedic impression of AECOPD or acute pulmonary edema; and 3) $\geq 12$ years old and $\geq 40 \mathrm{~kg}$ body weight. Contraindications to CPAP application were defined as Glasgow Coma Scale $<14$, facial anomalies, airway compromise, intubation/tracheostomy, respiratory rate $<8$ breaths per minute, blood pressure $<90 \mathrm{~mm} \mathrm{Hg}$, cardiac arrest, major trauma, or burns.

\section{Data collection}

Patient records were identified from the prehospital electronic database using the search criteria of presenting the complaint of "shortness of breath" and hospital return code "emergent." In addition to these criteria, all patients receiving prehospital CPAP in the after group were captured. An electronic copy of the prehospital patient record was obtained for each patient and was then linked to in-hospital records using name and date of birth as identifiers. Prehospital and in-hospital patient records were manually screened, and data were abstracted by the primary author. Those patients missing in-hospital data were not included in the analysis (Figure 1). Data points included patient demographics, times for EMS arrival and departure, time of CPAP application, criteria for CPAP application, ED and hospital lengths of stay, paramedic impression of diagnosis, prehospital intubation, ED intubation, final ED diagnoses, and survival to ED/ hospital discharge.

The use of "shortness of breath" as our initial search strategy was overly inclusive in our context, and the authors are confident that this captured all relevant patients. All use of CPAP was strictly documented, and the paramedic service assured $100 \%$ compliance.

\section{Outcome measures}

There were two main objectives in this study: 1) effectiveness and 2) safety. Each of these was defined with different suboutcomes, which will be explained here.

The primary effectiveness outcome was overall mortality. Secondary effectiveness outcomes were rates of intubation in the prehospital setting and the ED, rates of NIV in the ED, disposition from the ED, and length of stay.

For patient safety, the primary outcome was appropriateness of CPAP application based on the protocols to which the paramedics were trained. The secondary safety outcomes were rates of adverse events (paramedic reported adverse events as well as application of CPAP when contraindications were present) and the effect of CPAP on transport times. We included transport times in the safety arm of the study as a quality-control measure. We hypothesized that CPAP application would increase transport times and wanted to measure this to see whether it created an unreasonable delay.

There were no new co-interventions initiated during the study period. The use of symptom relief drugs was similar between groups.

\section{Data analyses}

Based on existing historical data, we predicted approximately 200 patients in the before and after 
groups. Sample size was primarily guided by feasibility in this unfunded study where we believed that two 3-month periods would provide sufficient patients to address the objectives. The association between the outcome measures and clinical variables was assessed by the appropriate univariate analyses according to the type of data: for nominal data, the $\chi^{2}$-test with continuity correction was used; for ordinal variables, the Mann-Whitney U-test was used; and, for continuous variables, the unpaired two-tailed $t$-test, using pooled or separate variance estimates as appropriate, was used.

\section{RESULTS}

We enrolled 341 patients from 383 charts reviewed, including 177 in the before CPAP group and 164 in the after CPAP group (see Figure 1). Ten patients in the before group and 22 in the after group had missing data, and thus were excluded from the analysis.

The mean age of patients was 71.5 years, with females comprising 51.4\%. Final diagnoses included
ACPE (18.9\%) and acute exacerbation of COPD (AECOPD) (21.9\%) (Table 1). Prehospital patient characteristics and transport times were similar between groups. Rates of EMS intubation were uniformly low, with only one intubation in the after group. There were a total of 36 CPAP applications in the after group. Paramedic diagnosis impressions differed between groups, with a higher rate of ACPE in the after group (34.6\% v. $18.2 \%$ ).

In-hospital patient characteristics for the before and after groups were similar, with lower oxygen saturations on presentation to the ED in the after group (93.4\% v. 99\%) (Table 2). Final ED diagnoses of ACPE and AECOPD were similar between groups. There was a higher incidence of pneumonia in the before group. Rates of NIV in the ED were higher in the after group $(20 \%$ v. $13.4 \%, p<0.0001)$, and there was no difference in the rates of intubation $(2.1 \%$ v. $2.3 \%, p<0.001)$. There was no statistically significant difference in length of stay $(6.8 \mathrm{v} .8 .7$ days, $p=0.24$ ). Disposition from the ED was similar between groups.

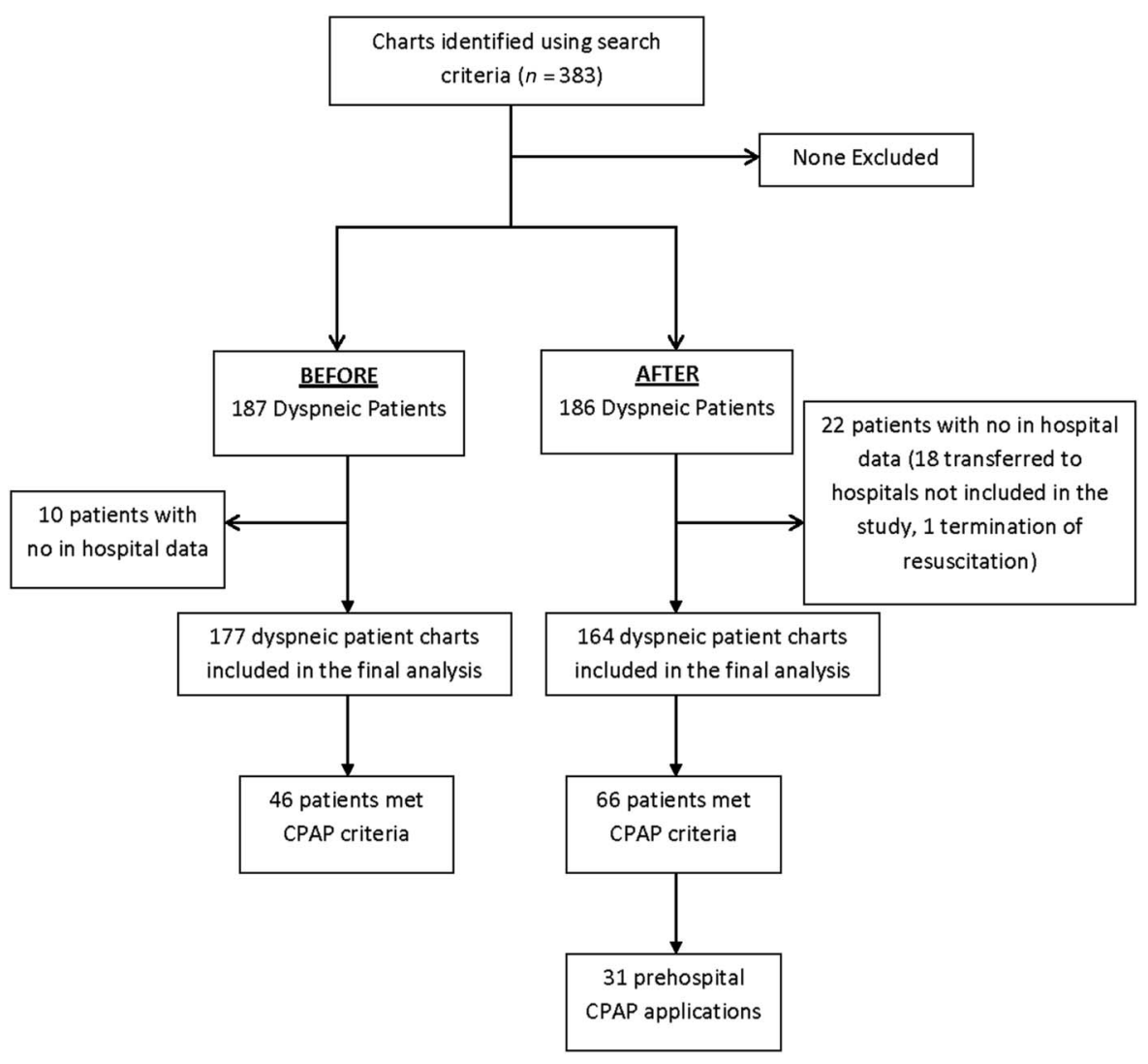

Figure 1. Patient Flow Diagram. 


\begin{tabular}{|c|c|c|}
\hline & Before $(n=177)$ & After $(n=164)$ \\
\hline Mean age in years (SD) & $70.6+/-17.1$ & $72.4+/-17.6$ \\
\hline Female sex (\%) & 52.4 & 50.5 \\
\hline \multicolumn{3}{|l|}{ EMS vital signs, mean } \\
\hline Temperature & 36.7 & 36.7 \\
\hline Oxygen saturation & 90.7 & 88.8 \\
\hline Respiratory rate & 29.7 & 30.2 \\
\hline Systolic blood pressure & 141.2 & 141.1 \\
\hline Heart rate & 105.2 & 104.6 \\
\hline EMS intubation, number & 0 & 1 \\
\hline EMS CPAP Applied, number & 0 & 36 \\
\hline \multicolumn{3}{|l|}{ EMS diagnosis impression (\%) } \\
\hline COPD & 19.7 & 20.7 \\
\hline $\mathrm{CHF}$ & 18.2 & 34.6 \\
\hline Dyspnea & 24.1 & 21.8 \\
\hline Other & 37.9 & 22 \\
\hline \multicolumn{3}{|l|}{ Transport Time Intervals (min) } \\
\hline Arrive scene - depart scene & 21.6 & 22.6 \\
\hline Arrive scene - apply CPAP & & 23.6 \\
\hline Depart scene - arrive ED & 10.1 & 9.6 \\
\hline
\end{tabular}

Analysis of the prehospital characteristics of only those patients meeting CPAP criteria $(n=112)$ yielded similar patient characteristics, although there were more female patients in the after group $(54.5 \% \mathrm{v}$. 45.6\%) (Table 3). Paramedic impressions varied between groups, with a greater proportion of AECOPD in the before group $(52.2 \%$ v. $33.3 \%)$ and a greater proportion of ACPE in the after group $(65.2 \% \mathrm{v}$. $43.5 \%$ ) (Tables 3 and 4).

There were a total of 31 EMS CPAP applications out of the 66 patients meeting application criteria in the after group (i.e., $53 \%$ of patients meeting CPAP criteria did not receive the intervention). Of the 98 patients not meeting CPAP criteria in the after group, 5 patients received the intervention (i.e., $5.4 \%$ of patients not meeting criteria received the intervention anyway) (Table 5). There were 12 minor adverse events in 36 applications $(33.3 \%)$, but none of these represented a patient safety issue (these patients did not tolerate CPAP in the prehospital setting).

In-hospital characteristics of only those patients meeting CPAP criteria were similar between groups. Rates of NIV in the ED were higher in the after group, but this was not statistically significant $(31.8 \%$ v. $21.7 \%$, $p=0.33$ ) (see Table 4). Of the patients who received prehospital CPAP, six of them had the intervention discontinued on arrival to the ED and did not receive

\begin{tabular}{|c|c|c|}
\hline & $\begin{array}{c}\text { Before } \\
(n=177)\end{array}$ & $\begin{array}{c}\text { After } \\
(n=164)\end{array}$ \\
\hline Mean age in years & $70.6+/-17.1$ & $72.4+/-17.6$ \\
\hline Female sex (\%) & 52.4 & 50.5 \\
\hline \multicolumn{3}{|l|}{ ED vital signs, mean } \\
\hline Temperature & 36.5 & 36.4 \\
\hline Oxygen saturation & 99 & 93.4 \\
\hline Respiration rate & 25.1 & 24.6 \\
\hline Systolic blood pressure & 141 & 136.8 \\
\hline Heart rate & 102.8 & 98.7 \\
\hline \multicolumn{3}{|l|}{ Final ED diagnosis (\%) } \\
\hline COPD & 22.9 & 20.9 \\
\hline $\mathrm{CHF}$ & 17.4 & 20.4 \\
\hline CHF or COPD & 2.1 & 2.2 \\
\hline Pneumonia & 28.8 & 19.9 \\
\hline Myocardial infarction & 3.2 & 3.2 \\
\hline Lung cancer & 4.3 & 4.8 \\
\hline Other & 20.9 & 28.4 \\
\hline \multicolumn{3}{|l|}{ ED interventions (\%) } \\
\hline Non Invasive Ventilation & 13.4 & 20 \\
\hline Intubation & 2.1 & 2.3 \\
\hline \multicolumn{3}{|l|}{ Disposition from ED (\%) } \\
\hline Discharged home & 29.3 & 23 \\
\hline Admitted to ward & 61.5 & 66.1 \\
\hline Admitted to ICU & 6.9 & 6.7 \\
\hline Died in ED & 2.1 & 4.3 \\
\hline $\begin{array}{l}\text { Average length of admission } \\
\text { (days) }\end{array}$ & 6.8 & 8.7 \\
\hline
\end{tabular}

NIV for the duration of their stay. Eleven patients had their NIV continued in-hospital. Twenty patients who received NIV in the ED had not received prehospital CPAP. We did not include this in the analysis because the delay in the latter group of patients receiving NIV was up to 20 hours, so their condition may have deteriorated and would not reflect what the paramedics assessed in the field. There was no statistically significant difference in intubation rates $(4.4 \%$ v. $1.5 \%$, $p=0.47)$.

There were significantly more patients discharged home from the ED in the before group (28.2\% v. $10.6 \%$, $p<0.05)$. Mortality in the ED was greater in the after group $(7.6 \%$ v. $0 \%, p<0.05)$. Length of stay in the ED was similar between groups, but the average length of admission was greater in the after group (12.2 days v. 6.6 days).

Overall mortality was greater in the after group (Figure 2, 18.8\% v. $14.9 \%, p<0.0001$ ). When looking at only those patients meeting CPAP criteria in each 


\begin{tabular}{|c|c|c|}
\hline & Before $(n=46)$ & After $(n=66)$ \\
\hline Mean age in years & 72.5 & 75.7 \\
\hline Female sex (\%) & 45.6 & 54.5 \\
\hline \multicolumn{3}{|l|}{ EMS vital signs, mean } \\
\hline Temperature & 36.5 & 36.6 \\
\hline Oxygen saturation & 89 & 86.4 \\
\hline Respiration rate & 31.9 & 31.7 \\
\hline Systolic blood pressure & 145.6 & 145.7 \\
\hline Heart rate & 107.4 & 102.9 \\
\hline EMS intubation, number & 0 & 1 \\
\hline EMS CPAP applied, number & 0 & 31 \\
\hline \multicolumn{3}{|l|}{ EMS impression (\%) } \\
\hline COPD & 52.2 & 33.3 \\
\hline $\mathrm{CHF}$ & 43.5 & 65.2 \\
\hline Dyspnea & 2.2 & 0 \\
\hline Other & 2.1 & 1.5 \\
\hline \multicolumn{3}{|l|}{ Transport time intervals (min) } \\
\hline Arrive scene - depart scene & 23.3 & 23.4 \\
\hline Arrive scene - apply CPAP & & 21.4 \\
\hline Depart scene - arrive ED & 10.7 & 9.1 \\
\hline
\end{tabular}

group, no statistically significant difference in mortality was observed (Figure 3, 19.7\% v. 10.9\%, $p=0.21$ ). There was one "do not resuscitate" (DNR) patient captured in the after group for those patients not surviving to hospital discharge. There were no DNR patients in the pre group for those patients not surviving to hospital discharge.

\section{DISCUSSION}

This before-and-after study was designed to assess the effectiveness of prehospital CPAP on patient mortality, morbidity, and length of stay. It was also designed to audit the safety and appropriateness of the application of this device by paramedics. We found the before and after groups to have similar patient characteristics, both prehospital and on presentation to the ED. CPAP did not show benefit with respect to rates of NIV in the ED, length of stay, or mortality. With respect to patient safety, there were very few applications of CPAP given the number of eligible patients, but no serious adverse events were reported as per protocol. It is unclear as to why there were so few applications of CPAP on patients who met clinical criteria. We did not include a questionnaire or debriefing tool for medics during this study, but, in speaking with paramedics and training staff, it would seem that the main barrier to

\begin{tabular}{|c|c|c|}
\hline & $\begin{array}{l}\text { Before } \\
(n=46)\end{array}$ & $\begin{array}{c}\text { After } \\
(n=66)\end{array}$ \\
\hline Mean age in years & 72.5 & 75.7 \\
\hline Female sex (\%) & 45.6 & 54.5 \\
\hline \multicolumn{3}{|l|}{ ED vital signs, mean } \\
\hline Temperature & 36.2 & 36.4 \\
\hline Oxygen saturation & 93.3 & 92.1 \\
\hline Respiration rate & 25.5 & 24.8 \\
\hline Systolic blood pressure & 142.3 & 136.7 \\
\hline Heart rate & 103.3 & 99.1 \\
\hline \multicolumn{3}{|l|}{ Final ED diagnosis (\%) } \\
\hline COPD & 41.3 & 34.8 \\
\hline $\mathrm{CHF}$ & 23.9 & 34.8 \\
\hline CHF or COPD & 6.5 & 3.0 \\
\hline Pneumonia & 13 & 18.1 \\
\hline Myocardial infarction & 4.3 & 4.5 \\
\hline Lung cancer & 2.2 & 4.5 \\
\hline Other & 13 & 4.5 \\
\hline \multicolumn{3}{|l|}{ ED interventions (\%) } \\
\hline Non Invasive ventilation & 21.7 & 31.8 \\
\hline Intubation & 4.4 & 1.5 \\
\hline \multicolumn{3}{|l|}{ Disposition from ED (\%) } \\
\hline Discharged home & 28.2 & 10.6 \\
\hline Admitted to ward & 65.2 & 74.2 \\
\hline Admitted to ICU & 6.5 & 7.6 \\
\hline Died in ED & 0 & 7.6 \\
\hline $\begin{array}{l}\text { Time to discharge/disposition from ED } \\
\text { (hours) }\end{array}$ & 4.8 & 6.5 \\
\hline Average length of admission (days) & 6.6 & 12.2 \\
\hline
\end{tabular}

Table 5. Number of Patients Meeting Criteria versus Actual Application of CPAP in the After Group

\begin{tabular}{llrr} 
& & \multicolumn{2}{c}{ Criteria Met } \\
\cline { 2 - 4 } & & Yes & No \\
\hline CPAP Applied & Yes & 31 & 5 \\
& No & 35 & 93 \\
\hline $\begin{array}{l}\text { Footnote: } 53 \% \text { of patients meeting criteria did not receive CPAP. 5.4\% of patients not } \\
\text { meeting criteria received CPAP. }\end{array}$
\end{tabular}

CPAP application was the perception by paramedics that a patient had to be sick enough to warrant the device.

As in our study, not all published reports have shown benefit. In a recent observational study by Cheskes et al., researchers were unable to demonstrate a decrease in intubation or mortality related to the use of prehospital CPAP. ${ }^{10} \mathrm{~A}$ recent historical cohort analysis by Aguilar et al. failed to show a decrease in intubation, ICU admission, length of stay, or mortality. ${ }^{11}$ 


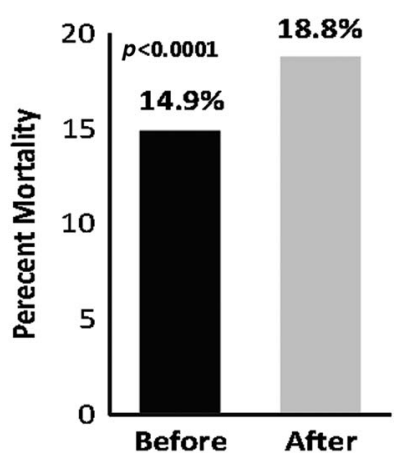

Figure 2. Overall Mortality Amongst the 321 Dyspnea Patients in the Before and After Groups.

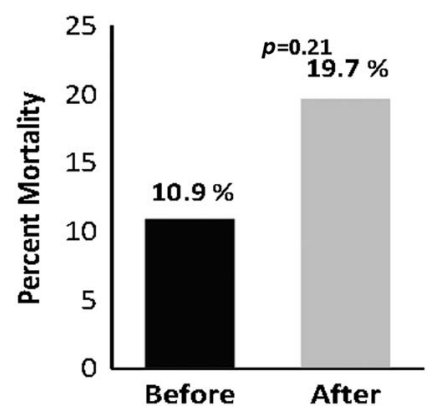

Figure 3. Mortality Amongst the 112 Patients Meeting CPAP Criteria in the Before and After Groups.

The results of this study are not consistent with several previous publications. Studies of this technology in the hospital setting have provided robust support for the routine use of NIV in patients with cardiogenic pulmonary edema and respiratory failure due to AECOPD. ${ }^{5,12}$ NIV has been shown to decrease rates of intubation, improve physiologic parameters, and even decrease mortality in hospital. ${ }^{6}$ Barriers to the widespread adoption of this treatment modality in the prehospital setting were largely due to the size, weight, and cost of older models; however, recent technological advances have led to increasing use. Furthermore, prehospital NIV has been shown to be an efficacious and cost-effective intervention for ACPE and AECOPD in several small prehospital studies. ${ }^{2,9}$ In a recent systematic review of randomized control trials by Mal et al., a significant reduction in mortality and need for invasive ventilation both in and out of the hospital was found. ${ }^{13}$ Williams et al. came to the same conclusion in their meta-analysis that included non-randomized and observational studies. ${ }^{14}$ Both of these reviews included primarily small studies, which presents a challenge to applicability to varied health care contexts.
Because of these positive findings, adoption of NIV is increasing exponentially, allowing for a growing body of prehospital evidence. As discussed by Williams et al. in their literature review, there is a significant deficit in high-quality research pertaining to non-invasive management of respiratory distress in the prehospital setting. ${ }^{14,15}$ NIV has been shown to be beneficial to patient care as a whole, but the question remains: Is the actual clinical impact of widespread prehospital CPAP worth the cost and training? How does it compare to other existing treatment modalities, such as nitrates, diuretics, and steroids?

This study has several strengths. First, it was a before-and-after study that measured the impact of this treatment modality in a real working environment, and there were no competing interventions during the study period. We conducted a comprehensive search of the database to capture all relevant patients and had balanced groups with respect to baseline patient characteristics. In order to minimize potential confounders, we allowed for a 3-month run-in period. We also selected identical months in 2 consecutive years to control for seasonal disease variations.

There were limitations to our study. The study was not blinded but this would be very difficult to accomplish. The study was neither multicentre nor randomized. We were missing some in-hospital data, and thus had to exclude a few patients in each group. Due to practical considerations, we limited our study to two EDs of one hospital, and thus limited our patient sample size. Data were collected by a single investigator, and, as such, we were not able to provide inter-rater reliability scoring (although the data extracted were objective). We also had a study population that was arguably less sick than in other studies; rates of intubation and use of NIV were low, which makes it difficult to draw conclusions from the data. Last, our centre is primarily urban with some rural areas, and thus our findings may not be generalizable to primarily rural populations.

\section{CONCLUSION}

Despite the robust in-hospital data supporting its use, we could not find benefit from CPAP in our prehospital setting with respect to morbidity, mortality, and length of stay. EMS must exercise caution in making the decision to invest in the equipment and training required to implement prehospital CPAP. 
Acknowledgements: We would like to thank the Ottawa Paramedic Service, Julie Sinclair (Regional Paramedic Program for Eastern Ontario), Dr. Michael Austin (The Ottawa Hospital), My-Linh Tran and Angela Marcantonio (Ottawa Hospital Research Institute).

Competing interests: None declared.

\section{REFERENCES}

1. Bruge $\mathrm{P}$, Jabre $\mathrm{P}$, Dru $\mathrm{M}$, et al. An observational study of noninvasive positive pressure ventilation in an out-ofhospital setting. Am 7 Emerg Med 2008;26(2):165-9.

2. Thompson J, Petrie DA, Ackroyd-Stolarz S, et al. Out-ofhospital continuous positive airway pressure ventilation versus usual care in acute respiratory failure: a randomized controlled trial. Ann Emerg Med 2008;52(3):232-41.

3. Peacock WF, De Marco T, Fonarow GC, et al. Cardiac troponin and outcome in acute heart failure. $N$ Engl $7 \mathrm{Med}$ 2008;358(20):2117-26.

4. Plant PK, Owen JL, Elliott MW. Early use of non-invasive ventilation for acute exacerbations of chronic obstructive pulmonary disease on general respiratory wards: a multicentre randomised controlled trial. Lancet 2000;355(9219):1931-5.

5. Gray AJ, Goodacre S, Newby DE, et al. A multicentre randomised controlled trial of the use of continuous positive airway pressure and non-invasive positive pressure ventilation in the early treatment of patients presenting to the emergency department with severe acute cardiogenic pulmonary oedema: the 3CPO trial. Health Technol Assess 2009;13(33):1-106.

6. Vital FM, Saconato H, Ladeira MT, et al. Non-invasive positive pressure ventilation (CPAP or bilevel NPPV) for cardiogenic pulmonary edema. Cochrane Database Syst Rev 2008:CD005351.

7. Girou E, Brun-Buisson C, Taille S, et al. Secular trends in nosocomial infections and mortality associated with noninvasive ventilation in patients with exacerbation of COPD and pulmonary edema. 7AMA 2003;290(22): 2985-91.

8. Levitt MA. A prospective, randomized trial of BiPAP in severe acute congestive heart failure. 7 Emerg Med 2001; 21(4):363-9.

9. Hubble MW, Richards ME, Wilfong DA. Estimates of costeffectiveness of prehospital continuous positive airway pressure in the management of acute pulmonary edema. Prehosp Emerg Care 2008;12(3):277-85.

10. Cheskes S, Turner L, Thomson S, et al. The impact of prehospital continuous positive airway pressure on the rate of intubation and mortality from acute out-of-hospital respiratory emergencies. Prehosp Emerg Care 2013;17(4):435-41.

11. Aguilar SA, Lee J, Dunford JV, et al. Assessment of the addition of prehospital continuous positive airway pressure (CPAP) to an urban emergency medical services (EMS) system in persons with severe respiratory distress. 7 Emerg Med 2013;45(2):210-9.

12. Tsai CL, Clark S, Cydulka RK, et al. Factors associated with hospital admission among emergency department patients with chronic obstructive pulmonary disease exacerbation. Acad Emerg Med 2007;14(1):6-14.

13. Mal S, McLeod S, Iansavichene A, et al. Effect of out-ofhospital noninvasive positive-pressure support ventilation in adult patients with severe respiratory distress: a systematic review and meta-analysis. Ann Emerg Med 2014;63(5): 600-7.

14. Williams TA, Finn J, Perkins GD, et al. Prehospital continuous positive airway pressure for acute respiratory failure: a systematic review and meta-analysis. Prehosp Emerg Care 2013;17(2):261-73.

15. Williams B, Boyme M, Robertson N, Giddings C. When pressure is positive: a literature review of the prehospital use of continuous positive airway pressure. Prehosp Disaster Med 2013;28(1):52-60. 